\title{
SAMPLE PATH LARGE DEVIATIONS OF POISSON SHOT NOISE WITH HEAVY-TAILED SEMIEXPONENTIAL DISTRIBUTIONS
}

\author{
KEN R. DUFFY, ${ }^{*}$ National University of Ireland, Maynooth \\ GIOVANNI LUCA TORRISI, ${ }^{* *}$ Istituto per le Applicazioni del Calcolo
}

\begin{abstract}
It is shown that the sample paths of Poisson shot noise with heavy-tailed semiexponential distributions satisfy a large deviation principle with a rate function that is insensitive to the shot shape. This demonstrates that, on the scale of large deviations, paths to rare events do not depend on the shot shape.
\end{abstract}

Keywords: Heavy-tailed distribution; sample path large deviation; Poisson shot noise

2010 Mathematics Subject Classification: Primary 60F10

\section{Introduction}

Shot noise processes have an extensive range of applications from physics [10], through electrical engineering [9] and queueing theory [4], [8]. They have also been used, for example, in risk theory to model the delay in claim settlement [5], [7]. It has recently been shown that Poisson shot noise (PSN) with independent and identically distributed (i.i.d.) heavy-tailed semiexponential shot values satisfies a scalar large deviation principle (LDP) with a rate function that is insensitive to the shot shape [11]. In this note we extend this result, proving that a sample path LDP holds for this process and, again, the resulting rate function is insensitive to the shot shape. The insensitivity manifests itself through the LDP having the same rate function as for a compound Poisson process with similarly distributed increments. Thus, on the scale of large deviations, the paths to rare events do not depend on the shot shape. The main result of this note can be viewed as the heavy-tailed counterpart of the sample path LDP for PSN under light tail conditions [4].

Our proof is inspired by Gantert's work on the centered partial sums of i.i.d. heavy-tailed semiexponential distributions [6]. The main novel difficulties stem from a lack of independent increments in PSN. These are overcome using the regenerative properties of the Poisson process in conjunction with delicate estimates to create a process with independent increments that is exponentially equivalent to that under study.

PSN is the following process:

$$
S(t)=\sum_{n=1}^{N(t)} H\left(t-T_{n}, Z_{n}\right), \quad t>0 .
$$

Received 18 January 2011; revision received 2 March 2011.

* Postal address: Hamilton Institute, National University of Ireland, Maynooth, Maynooth Co. Kildare, Ireland.

Email address: ken.duffy@nuim.ie

** Postal address: Istituto per le Applicazioni del Calcolo 'Mauro Picone' (IAC-CNR), Via dei Taurini 19, I-00185

Roma, Italia. Email address: torrisi@iac.rm.cnr.it 
Here $\{N(t)\}_{t>0}$ is a homogeneous Poisson process on $(0, \infty)$ with intensity $\lambda>0,\left\{T_{n}\right\}_{n \geq 1}$ are the points of the Poisson process, and $\left\{Z_{n}\right\}_{n \geq 1}$ form a sequence of i.i.d. random variables taking values in a measurable space $(E, \mathcal{E})$. The shot shape $H: \mathbb{R} \times E \rightarrow[0, \infty)$ is assumed measurable and is such that $H(t, z)=0$ for $t \leq 0$, and, for any $z \in E, H(t, z)$ is nondecreasing with respect to $t$. Throughout this paper, we assume that the sequences $\left\{T_{n}\right\}_{n \geq 1}$ and $\left\{Z_{n}\right\}_{n \geq 1}$ are independent, and denote by $H(\infty, z)$ the shot value, i.e. the limit of $H(t, z)$ as $t \rightarrow \infty$.

\section{Sample path large deviations}

We will prove that the sample paths of PSN satisfy the LDP in $D[0,1]$, the space of càdlàg functions defined on $[0,1]$, equipped with the $L_{1}$ topology induced by the norm $\|f\|=$ $\int_{0}^{1}|f(t)| \mathrm{d} t$. The idea is first to prove an LDP for the finite-dimensional distributions of the process and then lift this LDP to a principle for the process in $D[0,1]$ equipped with the topology of pointwise convergence using the Dawson-Gärtner theorem (see Theorem 4.6.1 of [3]). Finally, we strengthen the $L_{1}$ topology by demonstrating exponential tightness and establishing the upper and lower LDP bounds.

We begin by introducing basic definitions and recalling the scalar LDP for PSN with heavytailed semiexponential distributions as proved in [11]. We say that a family of random variables $\left\{V_{\alpha}\right\}_{\alpha>0}$ taking values in a topological space $(M, \tau)$ obeys an LDP with rate function $I$ and speed $v:[0, \infty) \mapsto[0, \infty)$ if $I: M \mapsto[0, \infty]$ is a lower semicontinuous function, $v$ is a measurable function such that $v(\alpha) \rightarrow \infty$ as $\alpha \rightarrow \infty$, and the following inequalities hold:

$$
\limsup _{\alpha \rightarrow \infty} \frac{1}{v(\alpha)} \log \mathrm{P}\left(V_{\alpha} \in C\right) \leq-\inf _{x \in C} I(x) \text { for all closed } C
$$

and

$$
\liminf _{\alpha \rightarrow \infty} \frac{1}{v(\alpha)} \log \mathrm{P}\left(V_{\alpha} \in O\right) \geq-\inf _{x \in O} I(x) \quad \text { for all open } O .
$$

Lower semicontinuity of $I$ means that its level sets, $\{x \in M: I(x) \leq c\}$ for $c \geq 0$, are closed. If the level sets are compact, the rate function $I$ is said to be good. The reader is referred to [3] for an introduction to large deviations theory.

We write $f(x) \sim g(x)$ if $f$ and $g$ are two nonnegative functions such that $f(x) / g(x) \rightarrow 1$ as $x \rightarrow \infty$ and, for a nonnegative random variable $X$, we define $\bar{F}(x)=\mathrm{P}(X>x)$, $x \geq 0$. Let $r \in(0,1)$ be a constant. We say that $\bar{F}$ or $X$ is heavy-tailed semiexponential if $\overline{\bar{F}}(x) \sim a(x) \exp \left\{-x^{r} L(x)\right\}$, where $a$ and $L$ are nonnegative slowly varying functions, i.e. $\lim _{x \rightarrow \infty} L(t x) / L(x)=1$ for all $t>0$ and the same holds for $a$. As is well known a semiexponential random variable $X$ has finite moments of all orders, but $\mathrm{E}\left[\mathrm{e}^{\theta X}\right]=\infty$ for all $\theta>0$. See, for example, [2] for an introduction to semiexponential distributions.

Theorem 2.1. ([11, Proposition 2.1].) Let $a$ and $L$ be positive slowly varying functions, let $r \in(0,1)$ be a positive constant, and define $\beta=\lambda \mathrm{E}[H(\infty, Z)]$. If

$$
\liminf _{t \rightarrow \infty} \frac{1}{t^{r} L(t)} \log \mathrm{P}(H(t, Z) \geq b t) \geq-b^{r} \text { for all } b>0
$$

and

$$
\mathrm{P}(H(\infty, Z) \geq t) \leq a(t) \exp \left\{-t^{r} L(t)\right\} \text { for all sufficiently large } t,
$$

then $\{S(t) / t\}_{t>0}$ obeys an LDP in $\mathbb{R}$ with speed $t^{r} L(t)$ and good, nonconvex rate function

$$
I^{(\beta)}(x)= \begin{cases}(x-\beta)^{r} & \text { if } x \geq \beta, \\ \infty & \text { if } x<\beta .\end{cases}
$$


Using this scalar LDP, we first prove that the LDP holds for finite-dimensional distributions. In doing so, we encounter the primary difficulty when compared to partial sums processes: the increments of PSN are not independent. This is overcome by the construction of an exponentially equivalent process with independent increments.

Theorem 2.2. Under the assumptions of Theorem 2.1, for any integer $k \geq 1$ and real numbers $0=t_{0}<t_{1}<t_{2}<\cdots<t_{k} \leq 1$, the family of random vectors $\left\{S\left(\alpha t_{1}\right) / \alpha, \ldots, S\left(\alpha t_{k}\right) / \alpha\right\}_{\alpha>0}$ satisfies an LDP in $\mathbb{R}^{k}$ with speed $\alpha^{r} L(\alpha)$ and good rate function

$$
I_{t_{1}, \ldots, t_{k}}^{(\beta)}\left(x_{1}, \ldots, x_{k}\right)=\sum_{i=1}^{k}\left(t_{i}-t_{i-1}\right)^{r} I^{(\beta)}\left(\frac{x_{i}-x_{i-1}}{t_{i}-t_{i-1}}\right),
$$

where $x_{0}=0$ and the function $I^{(\beta)}$ is defined in (2.1).

Proof. We divide the proof into five steps.

Step 1: an approximation with independent increments. Let $0=t_{0}<t_{1}<t_{2}<\cdots<t_{k} \leq 1$ be fixed. For $i=1, \ldots, k$, let $\left\{N^{(i)}(t)\right\}_{t>0}$ be i.i.d. copies of the Poisson process $\{N(t)\}_{t>0}$, and let $\left\{Z_{n}^{(i)}\right\}_{n \geq 1}$ be i.i.d. copies of the process $\left\{Z_{n}\right\}_{n \geq 1}$ that are independent of the Poisson processes $\left\{N^{(i)}(t)\right\}$. For $0<s \leq t \leq 1$ and $i=1, \ldots, k$, we define

$$
S^{(i)}(s, t)=\sum_{n=1}^{N^{(i)}(s)} H\left(t-T_{n}^{(i)}, Z_{n}^{(i)}\right) .
$$

By the regenerative property of the Poisson process and the i.i.d. property of the sequence $\left\{Z_{n}\right\}_{n \geq 1}$, the following equality in distribution holds:

$$
\begin{aligned}
& \left(S\left(t_{1}\right), S\left(t_{2}\right), \ldots, S\left(t_{k}\right)\right) \\
& \quad \stackrel{\mathrm{D}}{=}\left(S^{(1)}\left(t_{1}, t_{1}\right), S^{(1)}\left(t_{1}, t_{2}\right)+S^{(2)}\left(t_{2}-t_{1}, t_{2}-t_{1}\right), \ldots, S^{(1)}\left(t_{1}, t_{k}\right)\right. \\
& \left.\quad+S^{(2)}\left(t_{2}-t_{1}, t_{k}-t_{1}\right)+\cdots+S^{(k)}\left(t_{k}-t_{k-1}, t_{k}-t_{k-1}\right)\right) .
\end{aligned}
$$

For $\alpha>0$, set

$$
\begin{aligned}
\Sigma^{(1)}\left(\alpha, t_{1}, \ldots, t_{k}\right)=\alpha^{-1}( & \left.S\left(\alpha t_{1}\right), S\left(\alpha t_{2}\right)-S\left(\alpha t_{1}\right), \ldots, S\left(\alpha t_{k}\right)-S\left(\alpha t_{k-1}\right)\right), \\
\Sigma^{(2)}\left(\alpha, t_{1}, \ldots, t_{k}\right)=\alpha^{-1}( & S^{(1)}\left(\alpha t_{1}, \alpha t_{1}\right), S^{(2)}\left(\alpha\left(t_{2}-t_{1}\right), \alpha\left(t_{2}-t_{1}\right)\right) \\
+ & {\left[S^{(1)}\left(\alpha t_{1}, \alpha t_{2}\right)-S^{(1)}\left(\alpha t_{1}, \alpha t_{1}\right)\right], \ldots, } \\
& S^{(k)}\left(\alpha\left(t_{k}-t_{k-1}\right), \alpha\left(t_{k}-t_{k-1}\right)\right) \\
+ & \sum_{i=1}^{k-1}\left[S^{(i)}\left(\alpha\left(t_{i}-t_{i-1}\right), \alpha\left(t_{k}-t_{i-1}\right)\right)\right. \\
& \left.\left.-S^{(i)}\left(\alpha\left(t_{i}-t_{i-1}\right), \alpha\left(t_{k-1}-t_{i-1}\right)\right)\right]\right),
\end{aligned}
$$

and

$$
\begin{gathered}
\Sigma^{(3)}\left(\alpha, t_{1}, \ldots, t_{k}\right)=\alpha^{-1}\left(S^{(1)}\left(\alpha t_{1}, \alpha t_{1}\right), S^{(2)}\left(\alpha\left(t_{2}-t_{1}\right), \alpha\left(t_{2}-t_{1}\right)\right), \ldots,\right. \\
\left.S^{(k)}\left(\alpha\left(t_{k}-t_{k-1}\right), \alpha\left(t_{k}-t_{k-1}\right)\right)\right) .
\end{gathered}
$$


Step 2: exponential equivalence. Next we will show that the families of random vectors $\left\{\Sigma^{(2)}\left(\alpha, t_{1}, \ldots, t_{k}\right)\right\}_{\alpha>0}$ and $\left\{\Sigma^{(3)}\left(\alpha, t_{1}, \ldots, t_{k}\right)\right\}_{\alpha>0}$ are exponentially equivalent at the speed $\alpha^{r} L(\alpha)$. This claim follows if we can prove that, for fixed $s<t$, the following holds:

$\lim _{\alpha \rightarrow \infty} \frac{1}{\alpha^{r} L(\alpha)} \log \mathrm{P}\left(\sum_{n=1}^{N(\alpha s)}\left[H\left(\alpha t-T_{n}, Z_{n}\right)-H\left(\alpha s-T_{n}, Z_{n}\right)\right]>\alpha \delta\right)=-\infty$ for all $\delta>0$.

By the Chernoff bound we have, for any $\theta>0$,

$$
\begin{aligned}
& \mathrm{P}\left(\sum_{n=1}^{N(\alpha s)}\left[H\left(\alpha t-T_{n}, Z_{n}\right)-H\left(\alpha s-T_{n}, Z_{n}\right)\right]>\alpha \delta\right) \\
& \quad \leq \mathrm{e}^{-\theta \alpha \delta} \mathrm{E}\left[\exp \left\{\theta \sum_{n=1}^{N(\alpha s)}\left[H\left(\alpha t-T_{n}, Z_{n}\right)-H\left(\alpha s-T_{n}, Z_{n}\right)\right]\right\}\right] \\
& \quad=\mathrm{e}^{-\theta \alpha \delta} \exp \left\{\lambda \int_{0}^{\alpha s} \mathrm{E}\left[\exp \left\{\theta\left(H\left(\alpha t-u, Z_{1}\right)-H\left(\alpha s-u, Z_{1}\right)\right)\right\}-1\right] \mathrm{d} u\right\} .
\end{aligned}
$$

Therefore,

$$
\begin{aligned}
& \frac{1}{\alpha^{r} L(\alpha)} \log \mathrm{P}\left(\sum_{n=1}^{N(\alpha s)}\left[H\left(\alpha t-T_{n}, Z_{n}\right)-H\left(\alpha s-T_{n}, Z_{n}\right)\right]>\alpha \delta\right) \\
& \quad \leq-\frac{\theta \delta}{\left(L(\alpha) / \alpha^{1-r}\right)}+\frac{\lambda}{\alpha^{r} L(\alpha)} \int_{0}^{\alpha s} \mathrm{E}\left[\exp \left\{\theta\left(H\left(\alpha t-u, Z_{1}\right)-H\left(\alpha s-u, Z_{1}\right)\right)\right\}-1\right] \mathrm{d} u .
\end{aligned}
$$

Let $y>\beta$ be arbitrarily fixed. In step 5 we will show that if we take $\theta=d \alpha^{r-1} L(\alpha)$, with $0<d<(y-\beta)^{r-1}$, then

$$
\lim _{\alpha \rightarrow \infty} \frac{\lambda}{\alpha^{r} L(\alpha)} \int_{0}^{\alpha s} \mathrm{E}\left[\exp \left\{\theta\left(H\left(\alpha t-u, Z_{1}\right)-H\left(\alpha s-u, Z_{1}\right)\right)\right\}-1\right] \mathrm{d} u=0,
$$

so that

$$
\limsup _{\alpha \rightarrow \infty} \frac{1}{\alpha^{r} L(\alpha)} \log \mathrm{P}\left(\sum_{n=1}^{N(\alpha s)}\left[H\left(\alpha t-T_{n}, Z_{n}\right)-H\left(\alpha s-T_{n}, Z_{n}\right)\right]>\alpha \delta\right) \leq-d \delta
$$

and the claim follows by letting first $d$ tend to $(y-\beta)^{r-1}$ and then $y$ tend to $\beta$.

Step 3: large deviations for the family $\left\{\Sigma^{(3)}\left(\alpha, t_{1}, \ldots, t_{k}\right)\right\}_{\alpha>0}$. By Theorem 2.1 and the definition of the slowly varying function, we find that, for any fixed $t>0$, the stochastic process $\{S(\alpha t) /(\alpha t)\}_{\alpha>0}$ obeys an LDP on $\mathbb{R}$ with speed $\alpha^{r} L(\alpha)$ and good rate function $t^{r} I^{(\beta)}(x)$. Using the contraction principle (see Theorem 4.2.1 of [3]), we find that $\{S(\alpha t) / \alpha\}_{\alpha>0}$ obeys an LDP on $\mathbb{R}$ with speed $\alpha^{r} L(\alpha)$ and good rate function $t^{r} I^{(\beta)}(x / t)$. Owing to the independence of the processes $\left\{S^{(i)}(t, t)\right\}_{t>0}(i=1, \ldots, k)$, it follows from Exercise 4.2.7 of [3] that $\left\{\Sigma^{(3)}\left(\alpha, t_{1}, \ldots, t_{k}\right)\right\}_{\alpha>0}$ obeys an LDP on $\mathbb{R}^{k}$ with speed $\alpha^{r} L(\alpha)$ and good rate function

$$
\tilde{I}_{t_{1}, \ldots, t_{k}}^{(\beta)}\left(x_{1}, \ldots, x_{k}\right)=\sum_{i=1}^{k}\left(t_{i}-t_{i-1}\right)^{r} I^{(\beta)}\left(\frac{x_{i}}{t_{i}-t_{i-1}}\right) .
$$


Step 4: conclusion of the proof. By construction, $\Sigma^{(1)}\left(\alpha, t_{1}, \ldots, t_{k}\right) \stackrel{\mathrm{D}}{=} \Sigma^{(2)}\left(\alpha, t_{1}, \ldots, t_{k}\right)$ for all $\alpha>0$. Combining steps 2 and 3 with Theorem 4.2.13 of [3], we deduce that the family $\left\{\Sigma^{(1)}\left(\alpha, t_{1}, \ldots, t_{k}\right)\right\}_{\alpha>0}$ obeys an LDP on $\mathbb{R}^{k}$ with speed $\alpha^{r} L(\alpha)$ and good rate function $\tilde{I}_{t_{1}, \ldots, t_{k}}^{(\beta)}$ defined in (2.3). The claim follows by an application of the contraction principle with the function $\left(x_{1}, \ldots, x_{k}\right) \mapsto\left(x_{1}, x_{1}+x_{2}, \ldots, x_{1}+\cdots+x_{k}\right)$.

Step 5: proof of (2.2). All that remains is the establishment of the assertion in (2.2). Let $k \geq 1$ be an integer such that $r<k /(k+1)$, which exists as $r \in(0,1)$. By the inequality

$$
\mathrm{e}^{x}-1 \leq x+\frac{x^{2}}{2 !}+\frac{x^{3}}{3 !}+\cdots+\frac{x^{k+1}}{(k+1) !} \mathrm{e}^{x} \quad \text { for all } x \geq 0
$$

we have, for all $\alpha>0, t \geq s \geq 0$, and $0 \leq u \leq \alpha s$,

$$
\begin{aligned}
\frac{1}{\alpha^{r} L(\alpha)} & \mathrm{E}\left[\exp \left\{d \alpha^{r-1} L(\alpha)\left(H\left(\alpha t-u, Z_{1}\right)-H\left(\alpha s-u, Z_{1}\right)\right)\right\}-1\right] \\
\leq & \frac{d}{\alpha} \mathrm{E}\left[H\left(\alpha t-u, Z_{1}\right)-H\left(\alpha s-u, Z_{1}\right)\right] \\
& +\frac{1}{2} d^{2} \alpha^{r-2} L(\alpha) \mathrm{E}\left[H^{2}\left(\alpha t, Z_{1}\right)\right]+\cdots+\frac{1}{k !} d^{k} \alpha^{(k-1) r-k} L^{k-1}(\alpha) \mathrm{E}\left[H^{k}\left(\alpha t, Z_{1}\right)\right] \\
& +\frac{1}{(k+1) !} d^{k+1} \alpha^{k r-(k+1)} L^{k}(\alpha) \mathrm{E}\left[H^{k+1}\left(\alpha t, Z_{1}\right) \mathrm{e}^{d \alpha^{r-1} L(\alpha) H\left(\alpha t, Z_{1}\right)}\right] .
\end{aligned}
$$

So

$$
\begin{aligned}
\frac{1}{\alpha^{r} L(\alpha)} & \int_{0}^{\alpha s} \mathrm{E}\left[\exp \left\{d \alpha^{r-1} L(\alpha)\left(H\left(\alpha t-u, Z_{1}\right)-H\left(\alpha s-u, Z_{1}\right)\right)\right\}-1\right] \mathrm{d} u \\
\leq & \frac{d}{\alpha} \int_{0}^{\alpha s} \mathrm{E}\left[H\left(\alpha t-u, Z_{1}\right)-H\left(\alpha s-u, Z_{1}\right)\right] \mathrm{d} u \\
& +\frac{s}{2} d^{2} \alpha^{r-1} L(\alpha) \mathrm{E}\left[H^{2}\left(\alpha t, Z_{1}\right)\right]+\cdots+\frac{s}{k !} d^{k} \alpha^{(k-1)(r-1)} L^{k-1}(\alpha) \mathrm{E}\left[H^{k}\left(\alpha t, Z_{1}\right)\right] \\
& +\frac{s}{(k+1) !} d^{k+1} \alpha^{k(r-1)} L^{k}(\alpha) \mathrm{E}\left[H^{k+1}\left(\alpha t, Z_{1}\right) \mathrm{e}^{d \alpha^{r-1} L(\alpha) H\left(\alpha t, Z_{1}\right)}\right] .
\end{aligned}
$$

By the assumption on the distribution of $H\left(\infty, Z_{1}\right)$ we have $\mathrm{E}\left[H^{n}\left(\infty, Z_{1}\right)\right]<\infty$ for any $n \geq 1$, so that all the terms in the third line of the above inequality go to 0 as $\alpha \rightarrow \infty$. By a change of variable we deduce that

$$
\begin{aligned}
& \frac{d}{\alpha} \int_{0}^{\alpha s} \mathrm{E}\left[H\left(\alpha t-u, Z_{1}\right)-H\left(\alpha s-u, Z_{1}\right)\right] \mathrm{d} u \\
& \quad=d s \int_{0}^{1} \mathrm{E}\left[H\left(\alpha(t-z s), Z_{1}\right)-H\left(\alpha s(1-z), Z_{1}\right)\right] \mathrm{d} z
\end{aligned}
$$

and this latter term goes to 0 as $\alpha \rightarrow \infty$ by the dominated convergence theorem. Therefore, we only need to prove that

$$
\lim _{\alpha \rightarrow \infty} \alpha^{k(r-1)} L^{k}(\alpha) \mathrm{E}\left[H^{k+1}\left(\alpha t, Z_{1}\right) \mathrm{e}^{d \alpha^{r-1} L(\alpha) H\left(\alpha t, Z_{1}\right)}\right]=0 .
$$


Note that, for an arbitrary fixed $T>0$,

$$
\begin{aligned}
\mathrm{E}\left[H^{k+1}\right. & \left.\left(\alpha t, Z_{1}\right) \mathrm{e}^{d \alpha^{r-1} L(\alpha) H\left(\alpha t, Z_{1}\right)}\right] \\
= & \mathrm{E}\left[H^{k+1}\left(\alpha t, Z_{1}\right) \mathrm{e}^{d \alpha^{r-1} L(\alpha) H\left(\alpha t, Z_{1}\right)} \mathbf{1}\left\{H\left(\alpha t, Z_{1}\right)<T\right\}\right] \\
& +\mathrm{E}\left[H^{k+1}\left(\alpha t, Z_{1}\right) \mathrm{e}^{d \alpha^{r-1} L(\alpha) H\left(\alpha t, Z_{1}\right)} \mathbf{1}\left\{H\left(\alpha t, Z_{1}\right) \geq T\right\}\right] \\
\leq & T^{k+1} \mathrm{e}^{d \alpha^{r-1} L(\alpha) T}+\mathrm{E}\left[H^{k+1}\left(\alpha t, Z_{1}\right) \mathrm{e}^{d \alpha^{r-1} L(\alpha) H\left(\alpha t, Z_{1}\right)} \mathbf{1}\left\{H\left(\alpha t, Z_{1}\right) \geq T\right\}\right] .
\end{aligned}
$$

Since $\lim _{\alpha \rightarrow \infty} \alpha^{r-1} L(\alpha)=0$, the claim follows if we prove that

$$
\lim _{\alpha \rightarrow \infty} \alpha^{k(r-1)} L^{k}(\alpha) \mathrm{E}\left[H^{k+1}\left(\alpha t, Z_{1}\right) \mathrm{e}^{d \alpha^{r-1} L(\alpha) H\left(\alpha t, Z_{1}\right)} \mathbf{1}\left\{H\left(\alpha t, Z_{1}\right) \geq T\right\}\right]=0 .
$$

By the choice of $k \geq 1$ we have $k(r-1)+r(1+\varepsilon)^{-1}<0$ for all $\varepsilon>0$. An application of Hölder's inequality with conjugate exponents $(1+\varepsilon) / \varepsilon$ and $1+\varepsilon$ yields

$$
\begin{aligned}
\mathrm{E}\left[H^{k+1}\right. & \left.\left(\alpha t, Z_{1}\right) \mathrm{e}^{d \alpha^{r-1} L(\alpha) H\left(\alpha t, Z_{1}\right)} \mathbf{1}\left\{H\left(\alpha t, Z_{1}\right) \geq T\right\}\right] \\
\leq & \left(\mathrm{E}\left[H^{(k+1)(1+\varepsilon) / \varepsilon}\left(\alpha t, Z_{1}\right) \mathbf{1}\left\{H\left(\alpha t, Z_{1}\right) \geq T\right\}\right]\right)^{\varepsilon /(1+\varepsilon)} \\
& \times\left(\mathrm{E}\left[\mathrm{e}^{(1+\varepsilon) d \alpha^{r-1} L(\alpha) H\left(\alpha t, Z_{1}\right)} \mathbf{1}\left\{H\left(\alpha t, Z_{1}\right) \geq T\right\}\right]\right)^{1 /(1+\varepsilon)} .
\end{aligned}
$$

Note that

$$
\begin{gathered}
\left(\mathrm{E}\left[H^{(k+1)(1+\varepsilon) / \varepsilon}\left(\alpha t, Z_{1}\right) \mathbf{1}\left\{H\left(\alpha t, Z_{1}\right) \geq T\right\}\right]\right)^{\varepsilon /(1+\varepsilon)} \\
\quad \leq\left(\mathrm{E}\left[H^{(k+1)(1+\varepsilon) / \varepsilon}\left(\infty, Z_{1}\right)\right]\right)^{\varepsilon /(1+\varepsilon)} \in(0, \infty) .
\end{gathered}
$$

Thus, (2.4) follows if we show that

$$
\lim _{\alpha \rightarrow \infty} \alpha^{k(r-1)} L^{k}(\alpha)\left(\mathrm{E}\left[\mathrm{e}^{(1+\varepsilon) d \alpha^{r-1} L(\alpha) H\left(\alpha t, Z_{1}\right)} \mathbf{1}\left\{H\left(\alpha t, Z_{1}\right) \geq T\right\}\right]\right)^{1 /(1+\varepsilon)}=0 .
$$

This in turn follows if

$$
\limsup _{\alpha \rightarrow \infty} \frac{1}{\alpha^{r} L(\alpha)} \mathrm{E}\left[\mathrm{e}^{(1+\varepsilon) d \alpha^{r-1} L(\alpha) H\left(\alpha t, Z_{1}\right)} \mathbf{1}\left\{H\left(\alpha t, Z_{1}\right) \geq T\right\}\right]<\infty .
$$

Indeed (2.5) gives, for all large enough $\alpha$ and a positive constant $K_{1}>0$,

$$
\left(\mathrm{E}\left[\mathrm{e}^{(1+\varepsilon) d \alpha^{r-1} L(\alpha) H\left(\alpha t, Z_{1}\right)} \mathbf{1}\left\{H\left(\alpha t, Z_{1}\right) \geq T\right\}\right]\right)^{1 /(1+\varepsilon)} \leq K_{1} \alpha^{r /(1+\varepsilon)} L(\alpha)^{1 /(1+\varepsilon)} .
$$

Then

$$
\begin{aligned}
& \limsup _{\alpha \rightarrow \infty} \alpha^{k(r-1)} L^{k}(\alpha)\left(\mathrm{E}\left[\mathrm{e}^{(1+\varepsilon) d \alpha^{r-1} L(\alpha) H\left(\alpha t, Z_{1}\right)} \mathbf{1}\left\{H\left(\alpha t, Z_{1}\right) \geq T\right\}\right]\right)^{(1+\varepsilon)^{-1}} \\
& \quad \leq K_{1} \lim _{\alpha \rightarrow \infty} \alpha^{k(r-1)+r(1+\varepsilon)^{-1}} L^{k+(1+\varepsilon)^{-1}}(\alpha) \\
& \quad=0
\end{aligned}
$$

where the latter equality follows because $k(r-1)+r(1+\varepsilon)^{-1}<0$ and $L$ is slowly varying. In the remainder of the proof we establish the veracity of (2.5). Note that, if $X$ is a nonnegative random variable, $z>0$, and $0<U<\infty$, we have

$$
\mathrm{E}\left[\mathrm{e}^{z X} \mathbf{1}\{X \geq U\}\right] \leq \int_{U}^{\infty} z \mathrm{e}^{z s} \mathrm{P}(X>s) \mathrm{d} s+\mathrm{e}^{z U} \mathrm{P}(X \geq U)
$$


Then, for all large enough $\alpha$,

$$
\begin{aligned}
& \frac{\mathrm{E}\left[\mathrm{e}^{(1+\varepsilon) d \alpha^{r-1} L(\alpha) H\left(\alpha t, Z_{1}\right)} \mathbf{1}\left\{H\left(\alpha t, Z_{1}\right) \geq T\right\}\right]}{\alpha^{r} L(\alpha)} \\
& \quad \leq \frac{(1+\varepsilon) d}{\alpha} \int_{T}^{\infty} \mathrm{e}^{(1+\varepsilon) d \alpha^{r-1} L(\alpha) s} \mathrm{P}\left(H\left(\infty, Z_{1}\right)>s\right) \mathrm{d} s+\frac{\mathrm{e}^{(1+\varepsilon) d \alpha^{r-1} L(\alpha) T}}{\alpha^{r} L(\alpha)} .
\end{aligned}
$$

Therefore, for (2.5), it suffices to check that

$$
\limsup _{\alpha \rightarrow \infty} \int_{T}^{\infty} \frac{\mathrm{e}^{K_{2} \alpha^{r-1} L(\alpha) s}}{\alpha} \mathrm{P}\left(H\left(\infty, Z_{1}\right)>s\right) \mathrm{d} s<\infty \quad \text { for some } T>0,
$$

where we set $K_{2}=(1+\varepsilon) d$. Note that the sequence

$$
\limsup _{\alpha \rightarrow \infty} \int_{T}^{M} \frac{\mathrm{e}^{K_{2} \alpha^{r-1} L(\alpha) s}}{\alpha} \mathrm{P}\left(H\left(\infty, Z_{1}\right)>s\right) \mathrm{d} s, \quad M \geq 1,
$$

is nondecreasing with supremum

$$
\limsup _{\alpha \rightarrow \infty} \int_{T}^{\infty} \frac{\mathrm{e}^{K_{2} \alpha^{r-1} L(\alpha) s}}{\alpha} \mathrm{P}\left(H\left(\infty, Z_{1}\right)>s\right) \mathrm{d} s .
$$

Now, let $M \geq 1$ and $y>\beta$ be arbitrarily fixed and note that, for all $\alpha>M /(y-\beta)$,

$$
\begin{aligned}
\int_{T}^{M} \frac{\mathrm{e}^{K_{2} \alpha^{r-1} L(\alpha) s}}{\alpha} \mathrm{P}\left(H\left(\infty, Z_{1}\right)>s\right) \mathrm{d} s & \leq \int_{T}^{\alpha(y-\beta)} \frac{\mathrm{e}^{K_{2} \alpha^{r-1} L(\alpha) s}}{\alpha} \mathrm{P}\left(H\left(\infty, Z_{1}\right)>s\right) \mathrm{d} s \\
& \leq \int_{T}^{\infty} \frac{\mathrm{e}^{K_{2} \alpha^{r-1} L(\alpha) s}}{\alpha} \mathrm{P}\left(H\left(\infty, Z_{1}\right)>s\right) \mathrm{d} s .
\end{aligned}
$$

Taking first the limit as $\alpha \rightarrow \infty$ and then the limit as $M \rightarrow \infty$, we deduce that

$$
\begin{aligned}
& \limsup _{\alpha \rightarrow \infty} \int_{T}^{\infty} \frac{\mathrm{e}^{K_{2} \alpha^{r-1} L(\alpha) s}}{\alpha} \mathrm{P}\left(H\left(\infty, Z_{1}\right)>s\right) \mathrm{d} s \\
& \quad=\limsup _{\alpha \rightarrow \infty} \int_{T}^{\alpha(y-\beta)} \frac{\mathrm{e}^{K_{2} \alpha^{r-1} L(\alpha) s}}{\alpha} \mathrm{P}\left(H\left(\infty, Z_{1}\right)>s\right) \mathrm{d} s .
\end{aligned}
$$

We will show that, for large enough $T$,

$$
\limsup _{\alpha \rightarrow \infty} \int_{T}^{\alpha(y-\beta)} \frac{\mathrm{e}^{K_{2} \alpha^{r-1} L(\alpha) s}}{\alpha} \mathrm{P}\left(H\left(\infty, Z_{1}\right)>s\right) \mathrm{d} s=0,
$$

and (2.6) follows.

Proving (2.7) follows exactly as in Proposition 2.2 of [11] from Equation (18) to the end of the proof of part (ii). To make the current exposition self-contained, we provide these details here. For a fixed $r_{0} \in(0, r)$, by Theorem 1.5.4 of [1] we have $L(y) / y^{1-r} \sim \psi_{1}(y)$ and $a(y) / y^{r_{0}} \sim \psi_{2}(y)$, where $\psi_{1}$ and $\psi_{2}$ are nonincreasing functions. So, for any $\varepsilon^{\prime}>0$, there exists $y_{\varepsilon^{\prime}}$ such that, for all $y \geq y_{\varepsilon^{\prime}}$, we have

$$
\left(1-\varepsilon^{\prime}\right) \psi_{1}(y)<\frac{L(y)}{y^{1-r}}<\left(1+\varepsilon^{\prime}\right) \psi_{1}(y) \text { and } \frac{a(y)}{y^{r_{0}}}<\left(1+\varepsilon^{\prime}\right) \psi_{2}(y) .
$$


By assumption, the tail of $H\left(\infty, Z_{1}\right)$ is bounded above by $a(t) \exp \left\{-t^{r} L(t)\right\}$ for all large enough $t$, say for all $t \geq \bar{t}$. In the following we take $T>\max \left\{y_{\varepsilon^{\prime}}, \bar{t}\right\}$. By the upper bound on the tail of $H\left(\infty, Z_{1}\right), T>\bar{t}$, and the change of variable $z=s /[\alpha(y-\beta)]$, setting $K_{3}=K_{2}(y-\beta)$ we have

$$
\begin{array}{rl}
\int_{T}^{\alpha(y-\beta)} \frac{\mathrm{e}^{K_{2} \alpha^{r-1} L(\alpha) s}}{\alpha} \mathrm{P}\left(H\left(\infty, Z_{1}\right)>s\right) \mathrm{d} s \\
\leq(y-\beta) \int_{T /[\alpha(y-\beta)]}^{1} & a(z \alpha(y-\beta)) \\
& \times \exp \left\{K_{3} \alpha^{r} L(\alpha) z-(z \alpha)^{r}(y-\beta)^{r} L(z \alpha(y-\beta))\right\} \mathrm{d} z .
\end{array}
$$

Since $T>y_{\varepsilon^{\prime}}$, by (2.8) and the monotonicity of $\psi_{1}$, we have, for all $z \in(T /[\alpha(y-\beta)], 1)$,

$$
\frac{L(z \alpha(y-\beta))}{[z \alpha(y-\beta)]^{1-r}}>\frac{1-\varepsilon^{\prime}}{1+\varepsilon^{\prime}} \frac{L(\alpha(y-\beta))}{[\alpha(y-\beta)]^{1-r}},
$$

and so the right-hand side of (2.9) is less than or equal to

$$
\begin{array}{rl}
(y-\beta) \int_{T /[\alpha(y-\beta)]}^{1} & a(z \alpha(y-\beta)) \\
& \times \exp \left\{-K_{3} \alpha^{r} L(\alpha)\left[\frac{(y-\beta)^{r-1}}{K_{2}} \frac{1-\varepsilon^{\prime}}{1+\varepsilon^{\prime}} \frac{L(\alpha(y-\beta))}{L(\alpha)}-1\right] z\right\} \mathrm{d} z .
\end{array}
$$

By the choice of $d$ we can select $\varepsilon>0$ sufficiently small that $(y-\beta)^{r-1} / K_{2}>1$. Consequently, we can choose $\varepsilon^{\prime}$ sufficiently small that

$$
K_{4}=\left(\frac{1-\varepsilon^{\prime}}{1+\varepsilon^{\prime}}\right)^{2} \frac{(y-\beta)^{r-1}}{K_{2}}-1>0 .
$$

Since $L$ is slowly varying in correspondence of $\varepsilon^{\prime}$, there exists $t^{\prime}=t^{\prime}\left(y, \beta, \varepsilon^{\prime}\right)$ such that, for all $\alpha \geq t^{\prime}, L(\alpha(y-\beta)) / L(\alpha)>\left(1-\varepsilon^{\prime}\right) /\left(1+\varepsilon^{\prime}\right)$. Thus, using (2.10), we have

$$
\begin{aligned}
\int_{T}^{\alpha(y-\beta)} & \frac{\mathrm{e}^{K_{2} \alpha^{r-1} L(\alpha) s}}{t} \mathrm{P}\left(H\left(\infty, Z_{1}\right)>s\right) \mathrm{d} s \\
& \leq(y-\beta)\left(\sup _{z \in[T /(\alpha(y-\beta)), 1]} a(z \alpha(y-\beta))\right) \int_{T /[\alpha(y-\beta)]}^{1} \mathrm{e}^{-K_{5} \alpha^{r} L(\alpha) z} \mathrm{~d} z \\
& =K_{6}\left(\mathrm{e}^{-K_{7} \alpha^{r-1} L(\alpha)}-\mathrm{e}^{-K_{5} \alpha^{r} L(\alpha)}\right) \frac{\sup _{z \in[T /(\alpha(y-\beta)), 1]} a(z \alpha(y-\beta))}{\alpha^{r} L(\alpha)}
\end{aligned}
$$

for all large enough $\alpha$, where $K_{5}=K_{3} K_{4}, K_{6}=(y-\beta) / K_{5}$, and $K_{7}=K_{5} T /(y-\beta)$. Owing to the slow variation of $L, K_{6}\left(\mathrm{e}^{-K_{7} \alpha^{r-1} L(\alpha)}-\mathrm{e}^{-K_{5} \alpha^{r} L(\alpha)}\right)$ converges to $K_{6}$ as $\alpha \rightarrow \infty$. So (2.7) follows if

$$
\lim _{\alpha \rightarrow \infty} \frac{\sup _{z \in[T /(\alpha(y-\beta)), 1]} a(z \alpha(y-\beta))}{\alpha^{r} L(\alpha)}=0 .
$$

Since $T>y_{\varepsilon^{\prime}}$, by (2.8) and the monotonicity of $\psi_{2}$, we have, for all $z \in(T /[\alpha(y-\beta)], 1)$,

$$
a(z \alpha(y-\beta))<\left(1+\varepsilon^{\prime}\right)[z \alpha(y-\beta)]^{r_{0}} \psi_{2}(z \alpha(y-\beta)) \leq\left(1+\varepsilon^{\prime}\right)[\alpha(y-\beta)]^{r_{0}} \psi_{2}(T) .
$$


So

$$
\frac{\sup _{z \in[T /(\alpha(y-\beta)), 1]} a(z \alpha(y-\beta))}{\alpha^{r} L(\alpha)} \leq \frac{\left(1+\varepsilon^{\prime}\right)(y-\beta)^{r_{0}} \psi_{2}(T)}{\alpha^{r-r_{0}} L(\alpha)},
$$

and this latter term goes to 0 as $\alpha \rightarrow \infty$ owing to the slow variation of $L$ and the choice of $r_{0}$.

Armed with the finite-dimensional LDP in Theorem 2.2 we now complete the programme of proof by establishing that the sample path LDP holds in the topology of pointwise convergence and then in the $L_{1}$ topology. As a simple transformation of the rate function in question coincides with that for the centered partial sums of i.i.d. heavy-tailed semiexponential random variables, we can appeal to the results in [6] to assert its goodness.

Theorem 2.3. Under the assumptions of Theorem 2.1, the family $\{S(\alpha \cdot) / \alpha\}_{\alpha>0}$ obeys an LDP on $D[0,1]$ equipped with the topology of pointwise convergence with speed $\alpha^{r} L(\alpha)$ and good, nonconvex rate function

$$
J^{(\beta)}(f)= \begin{cases}\sum\left(f\left(t^{+}\right)-f\left(t^{-}\right)\right)^{r} & \text { if } f \in D_{\beta}[0,1], \\ \infty & \text { otherwise, }\end{cases}
$$

where the sum is taken over all the points of discontinuity of $f$ and

$D^{(\beta)}[0,1]=\{f \in D[0,1]: f$ is linearly increasing with slope $\beta$ between jumps,

which are nonnegative $\}$.

Proof. For $k \geq 1$, define the set of indexes

$$
\mathfrak{I}_{k}=\left\{\left(t_{1}, \ldots, t_{k}\right): t_{0}=0<t_{1}<\cdots<t_{k} \leq 1\right\} .
$$

By the Dawson-Gärtner theorem, it follows from Theorem 2.2 that $\{S(\alpha \cdot) / \alpha\}_{\alpha>0}$ obeys an LDP on $D[0,1]$ equipped with the topology of pointwise convergence with speed $\alpha^{r} L(\alpha)$ and good rate function

$$
\tilde{J}^{(\beta)}\left(f\left(t_{1}\right), \ldots, f\left(t_{k}\right)\right)=\sup _{k \geq 1,\left(t_{1}, \ldots, t_{k}\right) \in \mathfrak{I}_{k}} I_{t_{1}, \ldots, t_{k}}^{(\beta)}\left(f\left(t_{1}\right), \ldots, f\left(t_{k}\right)\right) .
$$

By the contraction principle with the map $f(t) \mapsto f(t)-\beta t,\{S(\alpha \cdot) / \alpha-\beta \cdot\}_{\alpha>0}$ satisfies an LDP in $D[0,1]$ equipped with the topology of pointwise convergence and a rate function $\tilde{J}^{(0)}$ as defined in (2.12). This rate function coincides with the rate function $I_{T}$ defined in [6] for the centered partial sums of i.i.d. heavy-tailed semiexponential distributions. In Lemma 4 of [6] it was established that $\tilde{J}^{(0)}$ coincides with $J^{(0)}$ ( $I$ on page 1358 of [6]) defined in (2.11). Thus, the identification of $\tilde{J}^{(\beta)}$ with the $J^{(\beta)}$ follows from another application of the contraction principle with the map $f(t) \mapsto f(t)+\beta t$. As this rate function mimics that found in [6], its goodness in the $L_{1}$ topology is proved in Lemma 8 there. The lack of convexity can be seen by noting that if $J^{(\beta)}(f)<\infty$ and $J^{(\beta)}(g)<\infty$, then, for any $\gamma \in(0,1)$,

$$
J^{(\beta)}(\gamma f+(1-\gamma) g)=\gamma^{r} J^{(\beta)}(f)+(1-\gamma)^{r} J^{(\beta)}(g)>\gamma J^{(\beta)}(f)+(1-\gamma) J^{(\beta)}(g) .
$$

In order to strengthen this LDP from the topology of pointwise convergence to the $L_{1}$ topology, we prove exponential tightness and use this property to directly prove that the upper and lower large deviation bounds hold in this topology. Exponential tightness alone is not sufficient to establish the LDP in the $L_{1}$ topology as, when equipped with the topology of 
pointwise convergence, $D[0,1]$ is not Hausdorff. For exponential tightness, we must establish the existence of compact sets $\left\{K_{L}\right\}_{L>0}$ in the $L_{1}$ topology such that

$$
\limsup _{\alpha \rightarrow \infty} \frac{1}{\alpha^{r} L(\alpha)} \log \mathrm{P}\left(\frac{S(\alpha \cdot)}{\alpha} \in K_{L}^{\mathrm{c}}\right) \leq-L,
$$

where $K_{L}^{\mathrm{c}}$ denotes the complement of $K_{L}$. For $L>0$, consider the sets

$$
K_{L}=\left\{f \in D[0,1]: \operatorname{var}_{[0,1]}(f) \leq L^{1 / r}+\beta\right\},
$$

where $\operatorname{var}_{[0,1]}(f)$ is the total variation of $f$ on $[0,1]$. Compactness of $K_{L}$ is shown in Lemma 5 of [6]. Note that

$$
\mathrm{P}\left(\frac{S(\alpha \cdot)}{\alpha} \in K_{L}^{\mathrm{c}}\right) \leq \mathrm{P}\left(\frac{S(\alpha)}{\alpha} \geq L^{1 / r}+\beta\right)
$$

and, thus, (2.13) follows from an application of Theorem 2.1. Using the sets $\left\{K_{L}\right\}_{L>0}$ again, note that, for any closed set $C$ in the $L_{1}$ topology, we have

$$
\mathrm{P}\left(\frac{S(\alpha \cdot)}{\alpha} \in C\right) \leq \mathrm{P}\left(\frac{S(\alpha \cdot)}{\alpha} \in C \cap K_{L}\right)+\mathrm{P}\left(\frac{S(\alpha \cdot)}{\alpha} \in K_{L}^{\mathrm{c}}\right) .
$$

As $C \cap K_{L}$ is closed in the topology of pointwise convergence, we can apply the LDP upper bound in that topology in addition to the identification of $\tilde{J}^{(\beta)}$ with $J^{(\beta)}$ and the exponential tightness to obtain the LDP upper bound

$$
\limsup _{\alpha \rightarrow \infty} \frac{1}{\alpha^{r} L(\alpha)} \log \mathrm{P}\left(\frac{S(\alpha \cdot)}{\alpha} \in C\right) \leq-\inf _{f \in C} J^{(\beta)}(f) .
$$

To prove the LDP lower bound,

$$
\liminf _{\alpha \rightarrow \infty} \frac{1}{\alpha^{r} L(\alpha)} \log \mathrm{P}\left(\frac{S(\alpha \cdot)}{\alpha} \in O\right) \geq-\inf _{f \in O} J^{(\beta)}(f)
$$

for any open set $O$ in the $L_{1}$ topology, it is enough to show that

$$
\liminf _{\alpha \rightarrow \infty} \frac{1}{\alpha^{r} L(\alpha)} \log \mathrm{P}\left(\frac{S(\alpha \cdot)}{\alpha} \in O_{\delta}(f)\right) \geq-J^{(\beta)}(f)
$$

for all $f$ such that $J^{(\beta)}(f)<\infty$ and

$$
O_{\delta}(f)=\left\{g: \int_{0}^{1}|f(t)-g(t)| \mathrm{d} t<\delta\right\} .
$$

For $n>0$, consider the following set:

$$
O^{(n)}(f)=\left\{g \text { nondecreasing: }\left|g\left(\frac{k}{n}\right)-f\left(\frac{k}{n}\right)\right|<\frac{1}{n} \text { for all } k \in\{1, \ldots, n\}\right\} .
$$

For sufficiently large $n, O^{(n)}(f) \subset O_{\delta}(f)$. To see this, define the intervals $I_{1}=[0,1 / n]$ and $I_{k}=((k-1) / n, k / n]$ for $k=2, \ldots, n$. As $g \in O^{(n)}(f)$ and $f$ and $g$ are nondecreasing, on the interval $I_{k}$ we have

$$
|f(t)-g(t)| \leq f\left(\frac{k}{n}\right)-f\left(\frac{k-1}{n}\right)+\frac{2}{n},
$$


and so

$$
\int_{I_{k}}|f(t)-g(t)| \mathrm{d} t \leq \frac{1}{n}\left(f\left(\frac{k}{n}\right)-f\left(\frac{k-1}{n}\right)\right)+\frac{2}{n^{2}} .
$$

Thus,

$$
\begin{aligned}
\int_{0}^{1}|f(t)-g(t)| \mathrm{d} t & =\sum_{k=1}^{n} \int_{I_{k}}|f(t)-g(t)| \mathrm{d} t \\
& \leq n \frac{2}{n^{2}}+\sum_{k=1}^{n} \frac{1}{n}\left(f\left(\frac{k}{n}\right)-f\left(\frac{k-1}{n}\right)\right) \\
& =\frac{2}{n}+\frac{1}{n}(f(1)-f(0)) .
\end{aligned}
$$

As the right-hand side is decreasing in $n$, we have $O^{(n)}(f) \subset O_{\delta}(f)$ for sufficiently large $n$. As $S(t)$ is nondecreasing almost surely, for sufficiently large $n$

$$
\mathrm{P}\left(\frac{S(\alpha \cdot)}{\alpha} \in O_{\delta}(f)\right) \geq \mathrm{P}\left(\frac{S(\alpha \cdot)}{\alpha} \in O^{(n)}(f)\right) .
$$

To prove the LDP lower bound and complete the proof, we note that, by Theorem 2.2, it follows that

$$
\begin{aligned}
& \liminf _{\alpha \rightarrow \infty} \frac{1}{\alpha^{r} L(\alpha)} \log \mathrm{P}\left(\frac{S(\alpha \cdot)}{\alpha} \in O^{(n)}(f)\right) \\
& \quad=\liminf _{\alpha \rightarrow \infty} \frac{1}{\alpha^{r} L(\alpha)} \log \mathrm{P}\left(\left|\frac{S(\alpha k / n)}{\alpha}-f\left(\frac{k}{n}\right)\right|<\frac{1}{n}, k \in\{1, \ldots, n\}\right) \\
& \quad \geq-\inf _{\left(x_{1}, \ldots, x_{n}\right) \in \prod_{k=1}^{n}(f(k / n)-1 / n, f(k / n)+1 / n)} I_{(1 / n, 2 / n, \ldots, 1)}^{(\beta)}\left(x_{1}, \ldots, x_{n}\right) \\
& \geq-\sum_{i=1}^{n}\left(\frac{1}{n}\right)^{r} I^{(\beta)}\left(n\left(f\left(\frac{i}{n}\right)-f\left(\frac{i-1}{n}\right)\right)\right) \\
& \geq-J^{\beta}(f) .
\end{aligned}
$$

\section{References}

[1] Bingham, N. H., Goldie, C. M. And Teugels, J. L. (1987). Regular Variation. Cambridge University Press.

[2] Borovkov, A. A. (2002). On subexponential distributions and the asymptotics of the distribution of the maximum of sequential sums. Sibirsk. Mat. Zh. 43, 1235-1264.

[3] Dembo, A. and Zeitouni, O. (1998). Large Deviations Techniques and Applications (Appl. Math. 38), 2nd edn. Springer, New York.

[4] Ganesh, A., Macci, C. And Torrisi, G. L. (2005). Sample path large deviations principles for Poisson shot noise processes, and applications. Electron. J. Prob. 10, 1026-1043.

[5] Ganesh, A. AND ToRrisi, G. L. (2006). A class of risk processes with delayed claims: ruin probability estimates under heavy tail conditions. J. Appl. Prob. 43, 916-926.

[6] Gantert, N. (1998). Functional Erdős-Renyi laws for semiexponential random variables. Ann. Prob. 26, 13561369.

[7] KlüPpelberg, C. AND Miкosch, T. (1995). Delay in claim settlement and ruin probability approximations. Scand. Actuarial J. 1995, 154-168.

[8] Konstantopoulos, T. and Lin, S.-J. (1998). Macroscopic models for long-range dependent network traffic. Queueing Systems 28, 215-243.

[9] Lowen, S. B. And Teich, M. C. (1990). Power-law shot noise. IEEE Trans. Inf. Theory 36, 1302-1318.

[10] Rice, S. O. (1944). Mathematical analysis of random noise. Bell System Tech. J. 23, 282-332.

[11] Stabile, G. and Torrisi, G. L. (2010). Large deviations of Poisson shot noise processes under heavy tail semi-exponential conditions. Statist. Prob. Lett. 2010, 1200-1209. 